\title{
A randomized trial of negative pressure wound therapy technology combined with intermittent instillation in the treatment of neck anastomotic leakage after esophageal cancer surgery
}

\author{
Bao-Jia Luo ${ }^{1 \#}$, Hui-Qin Zhang ${ }^{1 \#}$, Jiu-Di Zhong ${ }^{2}$, Xiang-Zi He ${ }^{3}$, Fang Shen ${ }^{4}$, Mei-Chun Zheng ${ }^{1}$, \\ Yong-Shan Wen ${ }^{1}$, Jin-Bo Li ${ }^{2}$, Ming-Zhu Xin ${ }^{5}$ \\ ${ }^{1}$ Colorectal Surgery Department, Sun Yat-sen University Cancer Center, State Key Laboratory of Oncology in South China, Collaborative \\ Innovation Center for Cancer Medicine, Guangzhou, China; ${ }^{2}$ Thoracic Surgery Department, Sun Yat-sen University Cancer Center, State Key \\ Laboratory of Oncology in South China, Collaborative Innovation Center for Cancer Medicine, Guangzhou, China; ${ }^{3}$ Traditional Chinese Medicine \\ and VIP Region, Sun Yat-sen University Cancer Center, State Key Laboratory of Oncology in South China, Collaborative Innovation Center for \\ Cancer Medicine, Guangzhou, China; ${ }^{4}$ Gastric Surgery Department, Sun Yat-sen University Cancer Center, State Key Laboratory of Oncology \\ in South China, Collaborative Innovation Center for Cancer Medicine, Guangzhou, China; ${ }^{5}$ Nursing Department, Sun Yat-sen University Cancer \\ Center, State Key Laboratory of Oncology in South China, Collaborative Innovation Center for Cancer Medicine, Guangzhou, China \\ Contributions: (I) Conception and design: BJ Luo, MC Zheng; (II) Administrative support: JD Zhong, XZ He, MZ Xin; (III) Provision of \\ study materials or patients: HQ Zhang, YS Wen; (IV) Collection and assembly of data: HQ Zhang, MC Zheng, F Shen; (V) Data analysis and \\ interpretation: BJ Luo; (VI) Manuscript writing: All authors; (VII) Final approval of manuscript: All authors. \\ "These authors contributed equally to this work. \\ Correspondence to: Ming-Zhu Xin. Sun Yat-sen University Cancer Center, 651 Dong Feng Road East, Yue-Xiu District, Guangzhou, China. \\ Email: xinmzh@sysucc.org.cn.
}

Background: This study aimed to examine the effects of applying the negative pressure wound therapy (NPWT) combined with intermittent instillation (NPWTi) in patients with cervical anastomotic leakage (AL) after esophageal cancer surgery.

Methods: From July 2019 to June 2021, 64 patients undergoing AL after esophageal cancer surgery were selected from our Hospital's Thoracic Department, and randomly allocated to the conventional nursing group (20 patients), the hospital central NPWTi group (23 patients), and the portable NPWTi group (21 patients). The hospital central NPWTi group was treated with central negative pressure combined with intermittent instillation, and the portable NPWTi group was treated with portable negative pressure combined with intermittent instillation. Indicators of fistula healing, healing days, treatment costs, comfort, and nursing satisfaction were examined in each group.

Results: The fistula healing rate, healing days, nursing satisfaction, and comfort level of the hospital central NPWTi group and the portable NPWTi group were better than those of the conventional nursing group $(\mathrm{P}<0.05)$. There was no difference in the fistula healing rates and healing days between the hospital central NPWTi group, and the portable NPWTi group $(\mathrm{P}>0.05)$. The treatment costs of the hospital central NPWTi group were lower than those of the portable NPWTi group $(\mathrm{P}<0.05)$.

Conclusions: Negative pressure treatment technology combined with the intermittent instillation of the neck anastomotic fistula improved the fistula microenvironment, strengthened the sterilization effect, drained the leachate effectively, shortened the fistula healing time, improved the fistula cure rate, and increased patients' satisfaction with nursing. In relation to the negative pressure source, there was no difference in the therapeutic effects of hospital central NPWTi compared to the portable negative pressure meter, but the hospital central NPWTi treatment costs were lower and patients' acceptance of NPWT instillation was higher. Thus, central NPWT instillation treatment is worthy of promotion.

$\wedge$ ORCID: 0000-0002-6357-9254. 
Trial Registration: Chinese Clinical Trial Registry ChiCTR2100052240.

Keywords: Neck anastomotic fistula; negative pressure wound therapy combined with intermittent instillation; nursing

Submitted Sep 03, 2021. Accepted for publication Nov 12, 2021.

doi: 10.21037/jgo-21-605

View this article at: https://dx.doi.org/10.21037/jgo-21-605

\section{Introduction}

Esophageal cancer is a common malignant tumor of the digestive tract in China (1). Currently, surgical resection is still the most effective radical treatment for esophageal cancer, especially early and mid-stage esophageal cancer (2). However, gastroesophageal anastomosis inevitably needs to be performed to reconstruct the digestive tract after removing the diseased esophagus. Among the complications following anastomosis, anastomotic leakage (AL) is not only the most common and serious complication after esophageal cancer surgery, but is also the main cause of surgical death (3-5). Notably, the incidence of neck AL is $4-30 \%$, and the mortality rate is up to $50 \%$ (6). Risk factors for anastomotic leak are systemic factors, such as malnutrition, hypotension, and hypoxia, and local factors, such as arterial or venous insufficiency, tension, infection, and technical problems. The etiology of anastomotic leak is often multifactorial (7). AL seriously affects patients' short-term quality of life and delays or suspends subsequent treatments, thereby reducing patients' long-term survival rates $(8,9)$.

The current clinical treatment of AL mainly comprises non-surgical symptomatic treatment, such as fasting and water prohibition, gastrointestinal decompression, adequate drainage, the insertion of a naso-intestinal tube for nutritional support, and anti-infection. The traditional treatment method for AL is routine dressing changes. As the amount of exudate, saliva, and secretions is large, the number of dressing changes required per day is large; however, this is very cumbersome and places a great burden on clinics. Patients also feel abnormal pain when the dressing is being changed, which increases their psychological burden and produces fear, which may in turn lead to a decrease in treatment compliance. Sticky secretions or residues often lead to poor drainage of the fistula, which may extend the infection to the thoracic cavity or mediastinum, causing the infection to persist and even become life threatening. The incidence of anastomotic stenosis remains about $10 \%$ even if the AL is completely healed (10). The key to treating neck anastomotic fistula is to maintain effective drainage. Thus, the question of how to continuously discharge sticky secretions and strong irritating digestive fluid to avoid siltation, promote the relative cleanliness of the anastomosis, and accelerate growth is the primary issue in the treatment of anastomotic fistula after esophageal cancer.

Negative pressure wound therapy (NPWT) is a new treatment that has emerged in recent years to promote wound healing. It combines traditional negative pressure attraction technology with closed dressings to remove the leachate and infection source of the wound (11). Continuous negative pressure prevents the spread of infection and protects the wound environment (11). It also produces a moist healing environment and stimulates the expression of a variety of healing-promoting genes and repair signals by promoting blood flow perfusion and granulation tissue to promote the synthesis and release of a variety of growth factors and enzymes around the wound surface, leading to epithelial regeneration (12). Additionally, NPWT effectively reduces edema around the wound tissue and enhance material exchange between the wound and around the wound to accelerate wound healing $(13,14)$. NPWT is effective in treating pressure ulcers, lower extremity ulcers, poor postoperative healing, diabetic foot, and other wounds $(15,16)$. However, very little research has been conducted on the application of NPWT in the treatment of fistula after head and neck tumors $(17,18)$. Previous studies have shown that the combination of NPWT and intermittent instillation (NPWTi) has certain advantages in the treatment of anastomotic fistula (19). The instillation fluid dilutes secretions and flushes out the dissolved necrotic tissues and bacteria to avoid blockages caused by excessive viscosity, and then combines with the negative pressure to suck out exudates and bacteria to control infection and promote healing (20). The overall healing time and speed of granulation crawling and epithelial localization of NPWTi were found to be better than those of applying NPWT only $(21,22)$.

The main source of negative pressure is central negative 
pressure and negative pressure treatment instruments (23). Notably, central negative pressure has high suction intensity, a low price, and is easy to obtain (16). However, central negative pressure limits the patient's activities to a certain extent, and fluctuation of pressure is easily affected by the utilization rate of the whole hospital. Negative pressure treatment instruments are portable, lightweight, and unaffected by patient activity during use; however, they are expensive and patients' acceptance of them is low. The 2 different negative pressure sources have their own advantages and disadvantages. At present, no research appears to have been conducted on which method provides better therapeutic effects and economic benefits. Thus, NPWTi was innovatively applied to the nursing of anastomotic fistula after esophageal cancer, and the therapeutic effect of NPWTi from two different sources of negative pressure were compared and analyzed to determine its economic benefits and clarify its application prospects in the treatment of cervical anastomotic fistula after esophageal cancer surgery. The results of this study provide a basis for the clinical popularization of use. We present the following article in accordance with the CONSORT reporting checklist (available at https://dx.doi.org/10.21037/ jgo-21-605).

\section{Methods}

\section{Design and the populations}

This single-center randomized trial comprised patients aged older than 18 years who were admitted to our hospital for radical esophageal cancer surgery and who developed cervical AL after surgery from July 2019 to June 2021. The study was approved by the Ethics Committee of the Sun Yat-sen University Cancer Center (approval number GYX2019-011).

To be eligible to participate in this study, patients had to meet the following inclusion criteria: (I) have been diagnosed with neck AL (for which symptoms include fever, chest pain, and chest tightness after surgery); (II) Display physical signs of AL, such as saliva-like fluid flowing out of the neck, which may be accompanied by a foul smell, palpation, and a feeling of a mixture of gas and liquid around the neck incision; and (III) have undergone a transesophageal barium swallow angiography that confirmed the occurrence of AL. Written informed consent was obtained from all patients.

Patients were excluded from the study if they met any of the following exclusion: (I) had thoracic fistula, mediastinal fistula, sepsis, multiple organ failure, a bleeding tendency, bleeding disorders, a mental disorder, or cognitive dysfunction after surgery; (II) had been discharged, died, or quit voluntarily after treatment for less than 7 days; (III) displayed poor compliance during the study period, or failed to comply or attempt to comply with the prescribed protocol; and/or (IV) had an adverse reaction or their condition worsened during the study, which led to their withdrawal from the study.

The trial is three-arm parallel study and the allocation ratio is close to $1: 1: 1$. Eighty patients with cervical anastomotic leakage (AL) after esophageal cancer surgery assessed for eligibility. In which, seventy-one patients met the inclusion and exclusion criteria and were randomly divided into groups through a table of random numbers for random grouping. Twenty patients were allocated to the conventional nursing group, 28 patients to the central NPWT instillation group, and 23 patients to the portable NPWT instillation group. During the course of treatment, 5 patients in the central NPWT instillation group (3 of whom who could not tolerate long-term bed rest, and 2 of whom who stopped treatment), and 2 patients in the portable NPWT instillation group (who stopped treatment due to economic reasons) withdrew halfway through the treatment. Ultimately, there were 20 patients in the conventional nursing group, 23 patients in the central NPWT instillation group, and 21 patients in the portable NPWT instillation group. No statistical differences were found in the general data of the 2 groups $(\mathrm{P}>0.05$; see Table 1$)$.

All procedures performed in this study involving human participants were in accordance with the Declaration of Helsinki (as revised in 2013).

\section{Intervention protocol}

\section{Conventional nursing}

Patients underwent the following procedures of conventional nursing, including: (I) Fasting and no water. (II) Gastrointestinal decompression (to aspirate acidic gastric juice, reduce gastroesophageal reflux, avoid gastric acid irritation to the anastomosis, and reduce the tension of the anastomosis). (III) Nutritional support (enteral nutrition was administered via a naso-intestinal tube to ensure an intake of 1,800-2,400 kcal each time. Parenteral nutrition was provided as necessary to promote the healing of the fistula as soon as possible). (IV) Anti-infection treatment (broad-spectrum and high-efficiency antibiotics were 
Table 1 Comparison of general information of the 3 groups

\begin{tabular}{lccc}
\hline Group & Age (years) & Fistula size $\left(\mathrm{cm}^{2}\right)$ & Time of fistula $(\mathrm{d})$ \\
\hline Conventional nursing group & $63.85 \pm 7.48$ & $1.05 \pm 1.15$ & $10.1 \pm 3.89$ \\
Hospital central NPWTi group & $62.89 \pm 7.40$ & $1.19 \pm 0.75$ & $10.36 \pm 3.09$ \\
Portable NPWTi group & $63.17 \pm 7.76$ & $1.70 \pm 1.19$ & $11.43 \pm 3.27$ \\
P & 0.909 & 0.115 & 0.421 \\
F & 0.096 & 2.244 & 0.879 \\
\hline
\end{tabular}

NPWT, negative pressure wound therapy; NPWTi, NPWT with intermittent instillation.
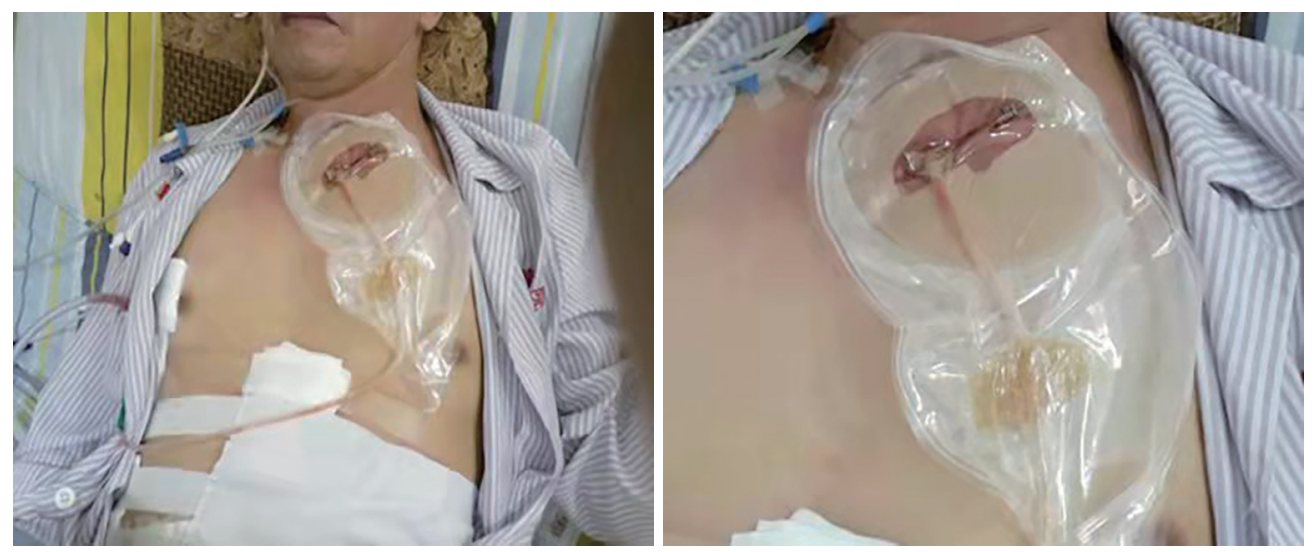

Figure 1 Treatment of neck anastomotic fistula using a stoma pouch.

used in the early stage, and sensitive antibiotics were then selected based on the results of a susceptibility test).

\section{Hospital central negative pressure combined with intermittent instillation (the central NPWTi group)}

The treatment comprised 5 steps. In Step 1, a double sleeve (a scalp needle hose) was inserted into the sputum suction tube. In Step 2, the wound was washed with saline and dried with dry square yarn. The front side holes of the double sets holes were coated with lipid hydrocolloid dressing, then tightened with surgical sutures and placed into $1 \mathrm{~cm}$ deep in the fistula. In Step 3, a 1-piece stoma pouch was sealed with an aperture $0.3 \mathrm{~cm}$ larger than the fistula. A thin colloid was attached and a "cross" opening was cut to the transparent pouch. The double sleeve was then worn through the chassis by the transparent pouch mouth and fixed with a colloidal seal (see Figure 1). In Step 4, the central negative pressure underwent continuous attraction, and the negative pressure was maintained at $125-150 \mathrm{mmHg}$ for over 22 hours a day. The dressing was replaced and the wound was observed every $2-3$ days. Next, normal saline $(30 \mathrm{~mL}$ of $0.9 \%$ normal saline each time) was used to wash the wound 4 times a day, and negative pressure after washing was maintained. Finally, the treatment costs and wound healing rates were evaluated according to the course of treatment (the course of treatment took 7 days).

\section{Negative pressure meter combined with intermittent} instillation group (the portable NPWTi group)

Steps 1 to step 3 were the same as those in the central NPWT instillation group (see above). However, in Step 4, a negative pressure meter was connected outside the suction tube using negative pressure drainage (Urgo Company, extriCARE2400). The negative pressure was maintained at $125-150 \mathrm{mmHg}$ for over 20 hours a day (It was suspended for 2 minutes every 5 minutes). Step 5 was the same as that used in the central NPWTi group (see above).

\section{Assessment criteria}

\section{Anastomotic granulation tissue coverage}

The wound tissue and surrounding skin were observed 
before treatment and at each dressing change. Redness and swelling, the volume, character and smell of secretions, changes in wound size and depth, and changes in new skin and granulation tissue were observed. Pictures were taken of the wound and granulation tissue areas on the surface of the neck fistula, which were also measured $(6,7)$. The coverage of anastomotic fistula granulation tissue on days 1, 3, 5, and 7 after the intervention was recorded. The calculation formula was as follows: granulation tissue coverage ratio $=$ granulation tissue area/wound area $\times 100.00$.

\section{Wound healing rate}

The following formula was used: (wound area before treatment - wound area after treatment)/wound area before treatment $\times 100 \%$ (20). The standard for wound cleaning was that the redness and swelling of wound tissue and surrounding skin subsided; the purulent discharge was well drained; the necrotic tissue and foreign bodies were basically cleared; the granulation tissue was fresh, had a fine granular texture, and bled easily when touched. The number of days it took to start negative pressure treatment to reach the cleansing of the wound was recorded.

\section{Anastomotic fistula healing time}

A gastrointestinal angiography was conducted to confirm that the anastomosis was completely healed. The following indicated that the anastomotic fistula had healed: the patient can eat, and there was no swelling or infection on the neck skin. The number of days it took to start the negative pressure treatment to heal was recorded.

\section{Negative pressure treatment costs}

Various consumables were required for NPWT, such as a suction tube, ostomy bag, irrigation fluid, and a negative pressure instrument. All the costs associated with negative pressure treatment were recorded.

\section{Feeling of comfort (21)}

The Visual analogue scale (VAS)pain score method was used to evaluate patients' overall comfort with the treatment after the treatment. Each patient was shown a straight line (100 $\mathrm{mm}$ in length) on which one end of the line was marked "0" (which represented "no comfort at all"), and the other end was marked "100" (which represented "maximum comfort"). Each patient marked the intensity of their own comfort feeling on the straight line, and the length from 0 to the marked point represented their comfort level.

\section{Nursing quality satisfaction}

The patient was asked to indicate their level of satisfaction with their overall treatment using a scale with the following 3 options: very satisfied, satisfied, or dissatisfied.

\section{Statistical analysis}

All the data were registered in an electronic database after being double-check by the researchers and analyzed using SPSS 22.0 software. The measurement data are expressed as mean \pm standard deviation. Independent sample $t$-tests were performed for both groups. The continuous variable factors were analyzed by a 1-way analysis of variance, and the categorical variables were analyzed using a chi-square analysis or Fisher's exact probability method. A P value $<0.05$ was considered statistically significant.

\section{Results}

\section{Participant flow}

Participants were randomly assigned and exclude for each group, please see the participant flow in detail (see Figure 2).

\section{Baseline data comparison}

Baseline data including patients' age, size of the fistula and the time fistula appeared after the surgery were compared between three groups and no statistical significance was found. It suggested that patients between three groups were comparable by statistical analysis $(\mathrm{P}>0.05$, see Table 1$)$.

\section{Coverage of anastomotic granulation tissue}

On the 1st day after the intervention, there was no difference in the coverage of anastomotic granulation tissue between the groups (see Table 2; $>>0.05$ ). Compared to the hospital conventional nursing group, the coverage of anastomotic granulation tissue in the central NPWTi group and the portable NPWTi group increased significantly on the $3 \mathrm{rd}, 5$ th, and 7 th days following the intervention (see Table 2; $\mathrm{P}<0.01$ ); however, there was no difference in the coverage of anastomotic granulation tissue between the central NPWTi group and the portable NPWTi group (see Table 2; $>>0.05)$. 


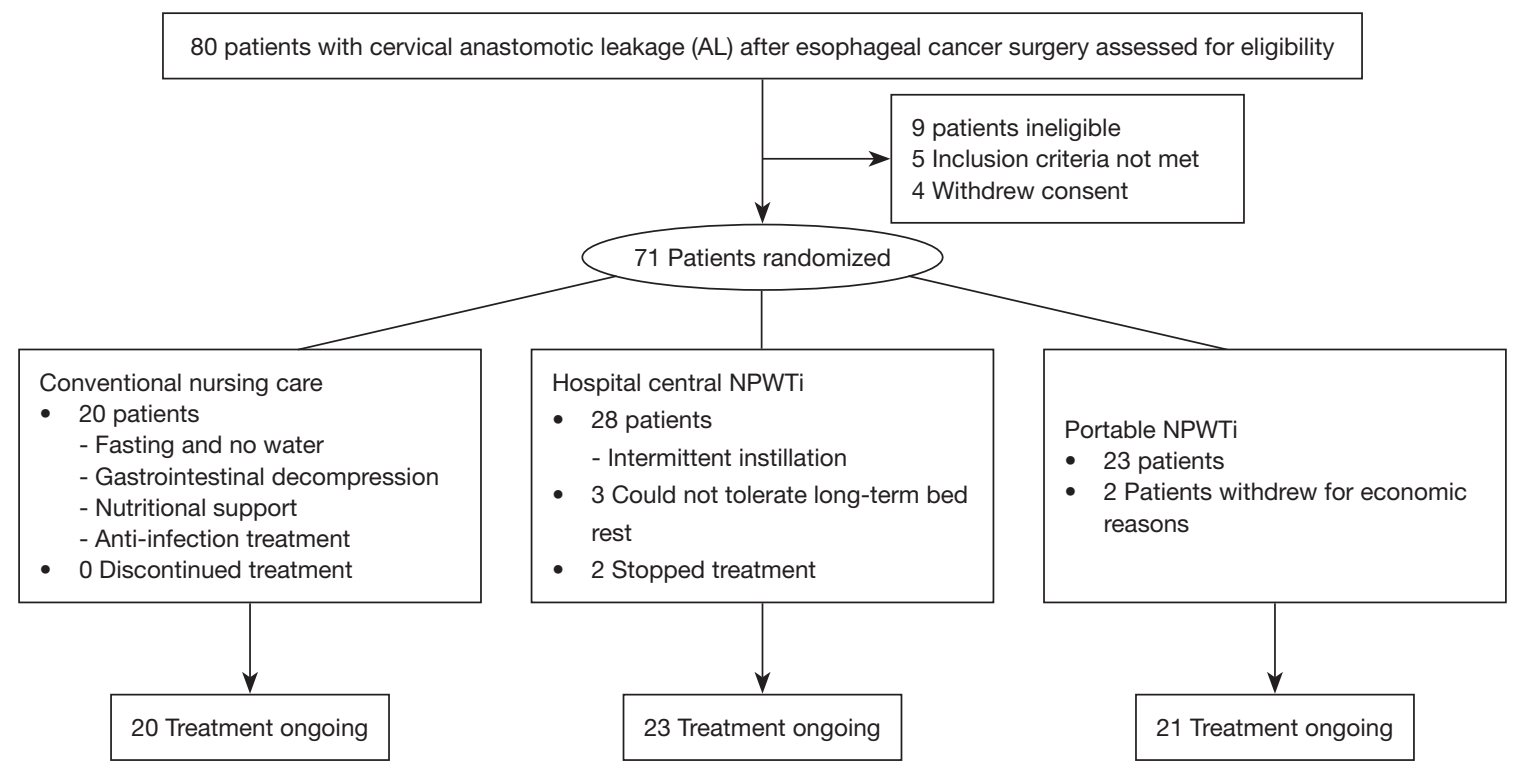

Figure 2 Participant flow of neck anastomotic fistula using a stoma pouch.

Table 2 Coverage of anastomotic granulation tissue.

\begin{tabular}{lcccc}
\hline Group & Day 1 & Day 3 & Day 5 & Day 7 \\
\hline Conventional nursing group & $2.87 \pm 0.38$ & $4.69 \pm 0.98$ & $6.53 \pm 1.41$ & $7.84 \pm 2.11$ \\
Hospital central NPWTi group & $3.31 \pm 0.82$ & $6.48 \pm 1.10$ & $8.19 \pm 1.88$ & $10.65 \pm 2.47$ \\
Portable NPWTi group & $3.43 \pm 1.02$ & $6.76 \pm 1.08$ & $8.48 \pm 1.48$ & 8.327 \\
P & 2.706 & 21.923 & $<0.01$ & 9.442 \\
F & 0.077 & $<0.01$ & $<0.01$ \\
\hline
\end{tabular}

NPWT, negative pressure wound therapy; NPWTi, NPWT with intermittent instillation.

\section{Wound bealing rate and average bealing time}

64 patients in this study all completed the wound treatment of AL. The cure rates of the hospital conventional nursing group, the central NPWTi group, and the portable NPWTi group were $55.00 \%$ (11/20), 82.61\% (19/23), and $85.71 \%(18 / 21)$, respectively, and the average healing times of these groups were $15.20 \pm 1.83$ days, $7.48 \pm 2.45$ days, and $7.19 \pm 1.89$ days, respectively. The cure rates of the 2 negative pressure treatment groups were significantly better than that of the conventional care group $(\mathrm{P}<0.01)$. The average healing times of the 2 negative pressure treatment groups were shorter than that of the conventional care group $(\mathrm{P}<0.05)$. However, there was no significant difference in the cure rates and average healing times between the two NPWT groups $(\mathrm{P}>0.05)$.

The average time for wound cleansing in the conventional nursing group was $11.05 \pm 2.31$ days, while the average times for wound cleansing in the central NPWTi group and the portable NPWTi group were $6.61 \pm 2.67$ days and 5.05 2.21 days, respectively. The average wound cleansing times of the two NPWT groups were significantly less than that of the hospital conventional nursing group $(\mathrm{P}<0.01)$. A comparison of different sources of negative pressure showed that the average time for wound cleaning in the central NPWTi group was significantly longer than that in the portable NPWTi group $(\mathrm{P}<0.05)$.

\section{Total length of hospitalization time and negative pressure treatment costs}

There were significant differences in the total hospitalization times and negative pressure treatment costs between the 3 
Table 3 Total length of hospitalization times and negative pressure treatment costs

\begin{tabular}{lcc}
\hline Group & Hospitalization time & Negative pressure treatment costs \\
\hline Conventional nursing group & $30.25 \pm 5.63$ & $14.20 \pm 0.77$ \\
Hospital central NPWTi group & $22.04 \pm 5.16$ & $12.88 \pm 0.67$ \\
Portable NPWTi group & $18.76 \pm 3.68$ & $17.75 \pm 0.92$ \\
P & $<0.05$ & $<0.05$ \\
F & 28.525 & 209.74 \\
\hline
\end{tabular}

NPWT, negative pressure wound therapy; NPWTi, NPWT with intermittent instillation.

groups (see Table 3; $\mathrm{P}<0.05$ ). The hospitalization times of the central NPWTi group and the portable NPWTi group were significantly shorter than that that of the conventional nursing group (see Table 3; $\mathrm{P}<0.05$ ). Notably, the portable NPWTi group had the shortest time (see Table 3; $\mathrm{P}<0.05$ ). A comparison of the different negative pressure sources showed that the central NPWTi group had a significantly longer hospitalization time than that of the portable NPWTi group (see Table 3; $\mathrm{P}<0.05$ ). In addition, the costs of the negative pressure treatment in the central NPWTi group were significantly lower than those of the hospital conventional nursing group and the portable NPWTi group (see Table 3; $\mathrm{P}<0.05$ ); the costs of the portable NPWTi group was the highest of the 3 groups.

\section{Comfort and satisfaction}

Patients in the central NPWTi group and the portable NPWTi group had higher comfort and nursing satisfaction levels compared to those of the conventional nursing group $(\mathrm{P}<0.05)$. The comfort and nursing satisfaction levels of patients in the portable NPWTi group were significantly higher than those of patients in the central NPWTi group $(\mathrm{P}<0.05)$.

\section{Discussion}

This study showed that the use of NPWT combined with intermittent instillation technology promptly and effectively removed necrotic tissue (based on the wound cleaning time), promoted wound granulation growth (based on the granulation tissue capping rate), shortened the healing time and the hospitalization time, improved the cure rate, increased patients' comfort (by preventing seepage, large saliva accumulation in the surface of wound, and cause impregnation), and improved patients' levels of satisfaction with the nursing they received. In terms of treatment costs, the central NPWTi group had the lowest treatment costs, while the portable NPWTi group had the highest treatment costs. The simple negative pressure materials, such as the 1-piece stoma pouch, disposable sputum suction tube, and simple negative pressure bottle, are relatively cheap and convenient to use. Conversely, portable negative pressure instruments, including the original matching piping and negative pressure conduction materials, are disposable and more expensive. The traditional treatment of cervical anastomotic fistula can easily cause a hypoxic environment of the wound that hinders the growth of new granulation tissues and prolongs the healing process (22). However, by applying NPWT technology, the wound exudate is removed, interstitial edema is decreased, the wound edges draw together, and the blood supply is promoted (23). Previous studies have shown that cell strain and the microdeformation of the wound promote the process of cell recruitment, proliferation, and differentiation, which in turn speed up healing (24).

In relation to the different sources of negative pressure, there was no significant difference in the wound granulation tissue capping rates, the healing rates, and the average healing times between the central NPWTi group and the portable NPWTi group. The wound cleaning time of the portable NPWTi group was significantly shorter than that of the central NPWTi group, while the hospitalization time of the central NPWTi group was significantly higher than that of the portable NPWTi group. The costs of the negative pressure treatment in the central NPWTi group were significantly lower than those of the conventional nursing group and the portable NPWTi group; the costs of the portable NPWTi group were the highest among the 3 groups. In addition, the levels of comfort and care satisfaction of patients in the portable NPWTi group was significantly higher than those of patients in the central NPWTi group. 
Traditional treatments for neck anastomotic leakage tend to cause a low oxygen environment around the wound's tissue, affecting the growth of new granulated tissue and delaying the wound healing cycle (25). For wound healing, oxygen pressure is the main control factor for sterilization, anti-infection, collagen synthesis, vascular hair and epithelial tissue formation (26). In the case of hypoxia, some or even all pathophysiological processes are affected. Negative pressure combined with the application of high concentration oxygen therapy increases local oxygen supply, It provides a physiological environment conducive to rapid tissue repair, which allows the creation of tissue to repair more quickly (27). In this study, the combination of hospital the two negative pressure treatment groups were significantly better than that of the conventional care group $(\mathrm{P}<0.01)$. The average healing times of the two negative pressure treatment groups were shorter than that of the conventional care group $(\mathrm{P}<0.05)$. It is shown that negative pressure wound therapy technology combined with intermittent instillation can continuously provide micro-flow pure oxygen, enhance the local oxygen separation of the wound surface, activate the phage function of inflammatory cells, promote the material and energy metabolism of the wound cells, and establish a closed wet healing microenfecture, thus accelerating the growth of granulated tissue and accelerating wound repair and healing. The rapid healing of the patient's fistula wound not only reduces the financial burden, but also reduces the workload of medical personnel and saves medical resources.

The portable device maximizes patient freedom and mobility, provides a single-patient-use NPWT solution for multi-week treatment that allows for the quick and easy discharge of patients from hospital to home, and reduces some of the logistical and administrative challenges associated with the provision of NPWT by caregivers (28). There was no difference between the central NPWTi group and the portable NPWTi group in terms of treatment effects. However, the portable negative pressure treatment instrument maintains a stable negative pressure, and provides a good sealing effect that can shorten the time to clean the wound surface by quickly promoting the decomposition and removal of the wound necrotic tissue. In terms of treatment costs, the overall central negative pressure treatment had the lowest treatment costs, and it also produced higher comfort and satisfaction levels among patients compared to conventional methods. The question of how to derive more economic benefits from the portable NPWT device requires a further analysis of more clinical cases. In the future, we hope that a more costeffective alternative treatment device will become available. However, there are some shortcomings, because the sample size selected in this study is small, and the conclusion has certain limitations, the sample size will be increased later for further study.

\section{Conclusions}

In this study, negative pressure treatment technology combined with intermittent instillation was found to effectively promote the healing of neck anastomotic fistula after esophageal cancer, shorten the time of hospitalization, promote patients' comfort and improve patients' satisfaction with nursing. In addition, the consumables used in the NPWT of the hospital central negative pressure source were low in price and easy to obtain. Hospital central NPWTi closely matches different types of pipes and materials, and thus is flexible to use. The overall treatment costs of hospital central NPWT were lower than those of the portable negative device; thus, it has more economic advantages. Further, it was more easily accepted for use, used more by clinical patients. However, the number of inspections should be increased to ensure the stable pressure of the central negative pressure device and the integrity of the pipeline to ensure the best therapeutic effects during temporary use.

\section{Limitations}

This study had some limitations. Each group comprised a relatively small number of patients, and the study was conducted at a single cancer center. Thus, the results should be interpreted cautiously, as the question of whether the Negative pressure stability is important for wound healing.

\section{Acknowledgments}

Funding: We received funding from the Guangdong Provincial Medical, Science and Technology Research Project 2019 (A2019239).

\section{Footnote}

Reporting Checklist: The authors have completed the CONSORT reporting checklist. Available at https://dx.doi. org/10.21037/jgo-21-605 
Trial Protocol: Available at https://dx.doi.org/10.21037/jgo21-605

Data Sharing Statement: Available at https://dx.doi. org/10.21037/jgo-21-605

Conflicts of Interest: All authors have completed the ICMJE uniform disclosure form (available at https://dx.doi. org/10.21037/jgo-21-605). The authors have no conflicts of interest to declare.

Ethical Statement: The authors are accountable for all aspects of the work in ensuring that questions related to the accuracy or integrity of any part of the work are appropriately investigated and resolved. All procedures performed in this study involving human participants were in accordance with the Declaration of Helsinki (as revised in 2013). The study was approved by the Ethics Committee of the Sun Yat-sen University Cancer Center (approval number GYX2019-011). Written informed consent was obtained from all patients.

Open Access Statement: This is an Open Access article distributed in accordance with the Creative Commons Attribution-NonCommercial-NoDerivs 4.0 International License (CC BY-NC-ND 4.0), which permits the noncommercial replication and distribution of the article with the strict proviso that no changes or edits are made and the original work is properly cited (including links to both the formal publication through the relevant DOI and the license). See: https://creativecommons.org/licenses/by-nc-nd/4.0/.

\section{References}

1. Chen $\mathrm{W}$, Zheng R, Baade PD, et al. Cancer statistics in China, 2015. CA Cancer J Clin 2016;66:115-32.

2. Mora A, Nakajima Y, Okada T, et al. Comparative study of predictive mortality scores in esophagectomy with threefield lymph nodedissection in patients with esophageal cancer. Dig Surg 2019;36:67-75.

3. Baiu I, Backhus L. Esophageal Cancer Surgery. JAMA 2020;324:1580.

4. Hoeppner J. Surgical strategy in multimodal treatment of gastric and esophageal cancer. Chirurg 2017;88:1005-9.

5. Moon SW, Kim JJ, Cho DG, et al. Early detection of complications: anastomotic leakage. J Thorac Dis 2019;11:S805-11.

6. Leenders BJ, Stronkhorst A, Smulders FJ, et al. Removable and repositionable covered metal self-expandable stents for leaks after upper gastrointestinal surgery: experiences in a tertiary referral hospital. Surg Endosc 2013;27:2751-9.

7. Sato S, Nakatani E, Higashizono K, et al. Size of the thoracic inlet predicts cervical anastomotic leak after retrosternal reconstruction after esophagectomy for esophageal cancer. Surgery. 2020;168:558-566.

8. Hua X, Qian R, Shi K, et al. Effectiveness and safety of bovine pericardium patch repair for cervical anastomotic leakage after oesophagectomy for cancer. J Thorac Dis 2019;11:3808-13.

9. Zhuo ZG, Li G, Deng HY, et al. Preoperative dilated esophagus is associated with a high risk of intrathoracic anastomotic leakage for patients with esophageal cancer. J Thorac Dis 2020;12:2325-32.

10. Chunwei F, Qingzeng N, Jianliang L, et al. Cervical esophagogastric anastomosis with a new stapler in the surgery of esophageal carcinoma. Eur J Cardiothorac Surg 2005;28:291-5.

11. Fleischmann W, Strecker W, Bombelli M, et al. Vacuum sealing as treatment of soft tissue damage in open fractures. Unfallchirurg 1993;96:488-92.

12. Broder KW, Nguyen B, Bodor RM. Negative Pressure Wound Therapy with Instillation in a Chronic NonHealing Right Hip Trochanteric Pressure Ulcer. Cureus 2016;8:e877.

13. Apelqvist J, Willy C, Fagerdahl AM, et al. EWMA Document: Negative Pressure Wound Therapy. J Wound Care 2017;26:S1-S154.

14. Lo Torto F, Ruggiero M, Parisi P, et al. The effectiveness of negative pressure therapy on infected wounds: preliminary results. Int Wound J 2017;14:909-14.

15. Cuomo R, Nisi G, Grimaldi L, et al. Use of ultraportable vacuum therapy systems in the treatment of venous leg ulcer. Acta Biomed 2017;88:297-301.

16. Raphael A, Gonzales J. Use of cryopreserved umbilical cord with negative pressure wound therapy for complex diabetic ulcers with osteomyelitis. J Wound Care 2017;26:S38-44.

17. Nguyen NA, Ringash J. Head and Neck Cancer Survivorship Care: A Review of the Current Guidelines and Remaining Unmet Needs. Curr Treat Options Oncol 2018;19:44.

18. Asher SA, White HN, Golden JB, et al. Negative pressure wound therapy in head and neck surgery. JAMA Facial Plast Surg 2014;16:120-6.

19. Goss SG, Schwartz JA, Facchin F, et al. Negative pressure wound therapy with instillation (NPWTi) better reduces 
post-debridement bioburden in chronically infected lower extremity wounds than NPWT alone. J Am Coll Clin Wound Spec. 2014;4:74-80.

20. Kim PJ, Attinger CE, Constantine T, et al. Negative pressure wound therapy with instillation: International consensus guidelines update. Int Wound J 2020;17:174-86.

21. Rycerz AM, Allen D, Lessing MC. Science supporting negative pressure wound therapy with instillation. Int Wound J 2013;10:20-4.

22. Fang JQ. Statistical methods for biomedical research. Higher Education Press 2007:283-303.

23. Yang MMH, Hartley RL, Leung AA, et al. Preoperative predictors of poor acute postoperative pain control: a systematic review and meta-analysis. BMJ Open 2019;9:e025091.

24. Rutegård M, Lagergren P, Rouvelas I, et al. Intrathoracic anastomotic leakage and mortality after esophageal cancer

Cite this article as: Luo BJ, Zhang $\mathrm{HQ}$, Zhong JD, He XZ, Shen F, Zheng MC, Wen YS, Li JB, Xin MZ. A randomized trial of negative pressure wound therapy technology combined with intermittent instillation in the treatment of neck anastomotic leakage after esophageal cancer surgery. J Gastrointest Oncol 2021;12(6):2665-2674. doi: 10.21037/jgo-21605 resection: a population-based study. Ann Surg Oncol 2012;19:99-103.

25. Xu J, Jiang QX, Liu Y, et al. A study on the effects of negative pressure wound therapy combined with topical oxygen therapy on orthopedic chronic wounds. Chinese Journal of Nursing 2016;51:650-654.

26. Yan S, Yue Y, Zeng L, et al. Ligustrazine nanoparticles nano spray's activation on Nrf2/ARE pathway in oxidative stress injury in rats with postoperative abdominal adhesion. Ann Transl Med 2019;7:379.

27. Song Z, Guo X, Zhang X. Effects of topical oxygen therapy on chronic traumatic wounds and its impact on granulation tissue. Am J Transl Res 2021;13:7294-9.

28. Glass GE, Nanchahal J. The methodology of negative pressure wound therapy: separating fact from fiction. J Plast Reconstr Aesthet Surg 2012;65:989-1001. 